\title{
La sobreexpresión del gen de enzima convertidora de angiotensina homóloga (ECA2) revierte la hipertensión arterial y el remodelado cardíaco experimental.
}

\author{
Elizabeth Ramos ${ }^{1}$, Alejandro Herrera², Jackeline Moya ${ }^{1}$, Felipe Apablaza', Iván Godoy ${ }^{1}$, Jorge Jalil', \\ Sergio Lavandero², Mario Chiong², María Paz Ocaranza'.
}

1 Facultad de Medicina. Pontificia Universidad Católica de Chile. 2 Centro FONDAP CEMC. Universidad de Chile.

Financiamiento: Fondecyt 1070662

\section{Resumen:}

Antecedentes: La sobreexpresión génica de la enzima convertidora de angiotensina I homóloga (ECA2) se asocia con prevención de la hipertrofia y fibrosis cardiaca dependiente de angiotensina (Ang) II. Sin embargo se desconoce si su sobreexpresión reduce la hipertensión arterial (HTA) y revierte el consecuente remodelado miocárdico (RM) dependiente de Ang II.

Objetivo: Determinar si la sobreexpresión adenoviral (Ad) del gen de la ECA2 en el miocardio disminuye la HTA y RM experimental en ratas con niveles genéticamente determinados de ECA y Ang II .

Métodos: Ratas homocigotas normotensas Lewis (LL) y Brown Borway (BN), con menores y mayores niveles circulantes de ECA y Ang II, respectivamente, se hicieron hipertensas por el procedimiento Goldblatt (GB). Como controles se usaron ratas seudo-operadas (sham). A la semana 5 post cirugía y con HTA establecida $\geq 140 \mathrm{mmHg}$, las ratas se randomizaron a inyección intramiocárdica con un AdECA2 o Ad proteína fluorescente verde (GFP) como controles de infección. A la semana post infección adenoviral, los ratas se sacrificaron y se determinaron peso corporal ( $\mathrm{PC}, \mathrm{g}$ ), masa cardiaca (MC, $\mathrm{mg}$ ), presión arterial sistólica (PAS, mmHg), área (AC, um2) y perímetro (PERC, um) de cardiomiocitos y contenido de colágeno (\%) miocárdico (CM), sub-endocárdico (CS) y total (CT).

Resultados: La HTA aumentó significativamente la MC, MCR, AC, PERC como también el CM, CS y CT en las ratas GB vs Sham, sin diferencias en el PC ni por efecto del polimorfismo de la ECA. La sobreexpresión de ECA2 disminuyó significativamente la PAS (15\% y $27 \%)$, AC ( $25 \%$ y $25 \%$ ) y PERC (17 \% y $18 \%)$ en las ratas $\mathrm{LL}$ y $\mathrm{BN}$ vs ratas hipertensas, respectivamente. Estos resultados se asociaron a una disminución significativa del CS ( $\mathrm{LL}=37 \%, \mathrm{BN}=39 \%), \mathrm{CM}(\mathrm{LL}=54 \%)$ y $\mathrm{CT}(\mathrm{LL}=42 \%, \mathrm{BN}=22 \%)$ respecto a las ratas $\mathrm{GB}$.

Conclusión: En ratas con HTA establecida, la sobreexpresión miocárdica de ECA2 disminuyó la HTA y el desarrollo de hipertrofia y fibrosis cardíaca hipertensiva experimental en ratas con diferentes niveles de ECA y Ang II. FONDECYT 1070662.

\section{Correspondencia:}

María Paz Ocaranza,

Departamento de

Enfermedades Cardiovasculares.

Facultad de Medicina.

P. Universidad Católica de Chile.

Tel: +562-3543407, Fax: +562-6321924.

E-mail: mocaran@med.puc.cl 


\section{Over expression of the homologous angiotensin converting enzyme (ACE2) gene reverts experimental hypertension and cardiac remodeling}

Objective: Background. Over expression of the gene for homologous angiotensin I converting enzyme (ACE) is associated with prevention of angiotensin II dependent cardiac hypertrophy and fibrosis. Whether this over expression is able to reduce hypertension and revert cardiac remodeling is unknown.

Aim: To determine whether adenoviral ACE2 gene over expression in the myocardium decreases experimental hypertension and cardiac remodeling in rats with genetically determined levels of ACE and ANG II.

Methods: Homozygous normotensive Lewis (LL) and

Brown Norway (BN) rats with decreased or increased levels of ACE and Ang II, respectively, were made hypertensive using the Goldblatt procedure. Sham operated rats were used as controls. 5 weeks after surgery, when systolic blood pressure reached $\geq 140 \mathrm{mmHg}$, rats were randomized to receive intramyocardial injection of either AdACE2 or green fluorescent Ad protein (GFP) as infection controlling agents. One week after

\section{Introducción:}

La hipertensión arterial (HTA) se define como un aumento sostenido de la presión arterial sistólica (PAS) sobre los $140 \mathrm{mmHg}$ y/o la presión diastólica (PD) sobre los $90 \mathrm{mmHg}^{1}$.

La HTA produce cambios tanto en la estructura como en la función de los órganos blanco, producto del remodelamiento miocárdico (RM). Son varios los cambios celulares y a nivel de tejido involucrados en el RM, como Hipertrofia ventricular, fibrosis miocárdica, inflamación y muerte celular ${ }^{2,3}$.

En la HTA hay varios mecanismos fisiopatológicos involucrados, siendo uno de ellos un desbalance en el Sistema Renina Angiotensina (SRA) ${ }^{4}$. Recientemente se han agregado nuevos componentes al SRA lo cual aumentó su complejidad en relación a la regulación en el balance de fluidos y de la presión arterial (PA) ${ }^{4}$.

La enzima convertidora de angiotensina I homóloga (ECA2) es un nuevo componente enzimático de la vía paralela del SRA ${ }^{5}$. Hoy en día se sabe que la ECA2 se expresa predominantemente en testículo, riñón y corazón, en células de músculo liso de vasos intra miocárdicos y en cardiomiocitos ${ }^{6}$. Presenta un $40 \%$ de adenoviral infection, rats were sacrificed. Body weight (BW, Gr), relative cardiac mass (RCM, mG), systolic blood pressure (SBP, mmHg), cardiomyocite area (MA um2) and perimeter (MPER, uM), and myocardial collagen content, both subendocardial (SC, \%) and total (TC \%) were measured.

Results: Hypertension was associated to significant increases in total and RCM, MA, MPER as well as SC and TC in Goldblatt vs normal rats. This was independent of BW and not affected by ACE polymorphism. ACE II over expression significantly decreased SBP (27 vs $15 \%$ ), MA (25 vs $25 \%$ ) and MPER (18 vs $17 \%$ ) hypertensive vs normal rats, respectively.

Conclusion: Over expression of ACE2 decreased hypertension, hypertrophy and fibrosis in rats with experimental hypertension and different levels of ACE and Ang II. (Fondecyt 1070662).

Key Words: ACE2, adenoviral infection, hypertension, cardiac hypertrophy.

homología con la secuencia aminoacídica con la ECA ${ }^{6}$ y difiere de esta última en la especificidad de sustrato y que no es inhibida por los inhibidores clásicos de la $\mathrm{ECA}^{7}$. La ECA2 es una carboxipeptidasa que forma Ang- ${ }^{1-9}$ a partir de Ang I y Ang- ${ }^{1-7}$ a partir de Ang II. Esta capacidad de regular los niveles de Ang I $^{1-7}$, como también el clearence de ang II ${ }^{7}$, hacen a la ECA2 blanco terapéutico para la regulación de la PA y de sus complicaciones ${ }^{8}$.

Varios estudios se han realizado en relación a rol de la ECA2 en la fisiología cardíaca 1, 5, 8, 9, 10,11, 12. La deficiencia de la ECA2 se ha asociado a daño cardíaco severo y aumento en los niveles plasmáticos y cardíacos de ang II ${ }^{8,9}$, situación que ha sido revertida en ratas knockout para ECA y ECA2 ${ }^{8}$. Estudios recientes han mostrado evidencia de que la sobreexpresión de la ECA2 a nivel del miocardio se asocia a prevención de la hipertrofia y fibrosis hipertensiva inducida por ang II, disminución en la producción de colágeno (col) I y III inducido por hipoxia en cultivo primario de fibroblastos ${ }^{10} \mathrm{y}$ protección de la función cardíaca y endotelial en isquemia coronaria permanente ${ }^{11}$. Todos los antecedentes disponibles en la literatura apuntan al rol preventivo de la ECA2 en la hipertrofia y fibrosis 
miocárdica inducida por Ang II.

La compleja inter-regulación entre ECA y ECA2 observada en ratas infartadas tratadas con enalapril

${ }^{13}$ hacen interesante evaluar el efecto de la sobre-expresión de ECA2 en un modelo genético en rata que expresa distintos niveles circulantes de ECA y Ang II. El modelo de ratas homocigotas Lewis (LL) y Brown Norway (BN) ha sido ampliamente utilizado en el Laboratorio ${ }^{14,15,16}$, describiendo que en condiciones basales, las ratas BN (modelo experimental similar a humanos con el alelo D y mayor actividad de ECA) ${ }^{13,14}$ presentan mayores niveles de ECA, renina, y Ang II ${ }^{15}$ pero baja actividad de NEP y Ang- ${ }^{1-7}{ }^{14}$. En cambio, las ratas LL presentan características opuestas [ $\left.{ }^{15}\right]$. A la fecha, no existen evidencias acerca de si la sobre expresión de la ECA2 es capaz de disminuir la HTA y regresar el remodelamiento miocárdico ya establecido. Por lo tanto, el objetivo de este estudio fue determinar si la sobreexpresión adenoviral del gen de la ECA2 a nivel del miocardio regresa HTA y el remodelamiento miocárdico hipertensivo experimental en ratas con niveles genéticamente determinados de ECA y Ang II.

\section{Materiales y Métodos:}

La presente investigación se realizó de acuerdo a la "Guía para el cuidado y uso de animales de laboratorio" publicado por el "National Health Institute" (NIH $\left.\mathrm{N}^{\circ} 85-23,1985\right)$ y fue aprobada por la Comisión de Investigación de la Facultad de Medicina de la Pontificia Universidad Católica de Chile.

1- Modelo experimental: Se utilizaron ratas homocigotas machos normotensas (peso $150 \pm 10 \mathrm{~g}$ ) de segunda generación (F2), producto de la cruza de ratas normotensas de las cepas Brown Norway (BB) y Lewis (LL), las cuales presentan un polimorfismo del gen ECA ${ }^{12}$. La selección de las ratas homocigotos BN y LL se realizó mediante la amplificación por PCR del fragmento de DNA que contiene el polimorfismo 13 .

Las ratas fueron randomizadas y la hipertensión experimental fue inducida usando el modelo Goldblatt $(\mathrm{Gb}$; $2 \mathrm{r}-1 \mathrm{p}$ ) $\left.{ }^{[15}\right]$. Como controles (Sham) se usaron animales seudo operados. Cinco semanas post cirugía, las ratas con HTA establecida $>140 \mathrm{mmHg}$ fueron randomizadas a infección intra-miocárdica tal como lo describe Hajjar et al. $\left[{ }^{17}\right]$ con un vector adenoviral que sobre expresa la ECA2 (AdECA2) o la proteína fluorescente verde (GFP). A la semana después de la infección, las ratas fueron eutanasiadas.
2- Parámetros hemodinámicos: La PA fue medida semanalmente por pletismografía en la cola de las ratas previamente anestesiadas.

3- Obtención del tejido ventricular: Los animales fueron anestesiados con Ketamina $(35 \mathrm{mg} / \mathrm{kg})$ y Xilacina $(7 \mathrm{mg} / \mathrm{kg})$ por vía intra-peritoneal. El miocardio fue extraído y lavado en suero fisiológico, una parte del ventrículo fue fijado en bouin por 12 horas y posteriormente incluido en parafina. El resto del tejido fue conservado a $-80^{\circ} \mathrm{C}$.

4- Evaluación de la hipertrofia cardíaca: Se usaron como parámetros de hipertrofia la masa cardíaca (MC), la masa cardíaca relativa (MCR, masa cardía$\mathrm{ca} /$ masa corporal $* 100$ ). Junto con, el tamaño y perímetro de los cardiomiocitos. Para esto de una sección de ventrículo, previamente incluida en parafina, se realizaron cortes de $5 \mu \mathrm{m}$ de grosor, que fueron sometidos a tinción con hematoxilina-eosina ${ }^{18}$. Los cortes fueron examinados bajo microscopio de luz (Nikon), las imágenes fueron captadas mediante una cámara Nikon DS Fi1 y proyectadas a un monitor mediante un software. Se midió y registró el área y perímetro de los cardiomiocitos, utilizando el programa Nis-Element.

5- Evaluación del desarrollo de fibrosis en el miocardio por morfometría: Se utilizaron cortes de 5 $\mu \mathrm{m}$ de ventrículo, previamente incluido en parafina, los cuales fueron teñidos con rojo picrosirio. El tejido fue examinado bajo microscopio de luz, las imágenes fueron captadas con cámara Nikon y proyectadas a un monitor. Se utilizó el programa Matlab, con el cuál se midió el contenido de colágeno intersticial según el procedimiento descrito por Ocaranza et al ${ }^{16}$.

6- Análisis de resultados: Los datos obtenidos se expresaron como promedio \pm S.E.M. Los grupos experimentales estuvieron constituidos por 7-10 animales. Para las comparaciones se usó análisis estadístico con ANOVA seguido de prueba de $t$ de Student- NewmanKeuls. El análisis estadístico se realizó usando el programa estadístico SPSS 10.0. Un valor de $\mathrm{p}<0,05$ fue considerado como estadísticamente significativo.

\section{Resultados:}

\section{Peso corporal, presión arterial y masa cardíaca relativa.}

El PC en las ratas AdECA2 LL y BN fue significativamente menor $(-21 \%$ y $-6 \%)$ respecto a las ratas $\mathrm{GB}$ y sham LL y BN. No se observaron diferencias en el 


\begin{tabular}{lccccccccc}
\multicolumn{6}{c}{ Tabla 1: Presi } & \multicolumn{7}{c}{ n arterial sist lica y masa card aca relativa de ratas } \\
hipertensas con polimorfismo de la ECA e infectadas con AdECA2.
\end{tabular}

Los resultados se presentan como promedio \pm SEM. Abreviaciones PC: peso corporal (g), PAS: presión arterial sistólica $(\mathrm{mmHg}), \mathrm{MC}$ : masa cardiaca $(\mathrm{mg}), \mathrm{MCR}$ : masa cardiaca relativa $(\mathrm{MC}(\mathrm{g}) / \mathrm{PC}(\mathrm{g}) x$ 100). *p<0.05 vs $S, G B, \# p<0.05$ vs $S, \& p<0.05$ vs $G B, \$ p<0.05$ vs AdECA2

PC entre las ratas AdECA2 y AdGFP (Tabla 1).

La PAS no mostró diferencias entre las ratas normotensas LL-S como BN-S, durante todo el estudio (tabla 1). En las ratas GB LL y BN se observó un aumento significativo de la PAS (42\%) desde la primera semana post cirugía hasta la $6^{\circ}$ semana. La infección con AdECA2 disminuyó significativamente la PAS $(-15 \%,-27 \%)$ para los grupos LL y BN, respectivamente, respecto a las ratas GB a la semana post infección. Mientras que los grupos AdGFP LL y BN mostraron una disminución significativa de la PAS (- 22\%), sin diferencias por efecto del polimorfismo de la ECA (Tabla 1).

La MC en la ratas GB fue significativamente mayor $(943 \pm 41$ vs $740 \pm 20$ en LL y $881 \pm 21$ vs $792 \pm 31$ en BN) como también la MCR $(0.387 \pm 0.02$ vs $0.334 \pm 0.01$

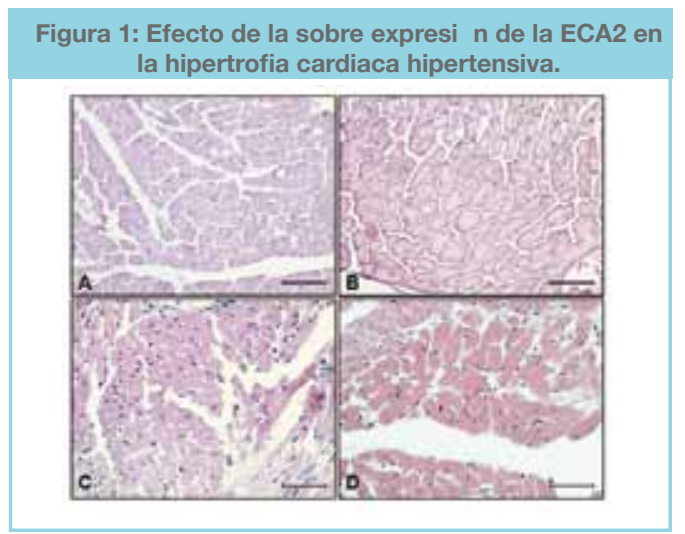

Luego de una semana de la infección con el vector adenoviral, el ventrículo fue extraído, tratado, cortado y medido tal como se describe en materiales y método. La imagen representa los cardiomiocitos de ratas Sham (A), Goldblatt (B), Goldblatt tratadas con AdECA2 (C) y Goldblatt tratadas con AdGFP (D). La barra equivale a $50 \mu \mathrm{m}$. Fotos tomadas en $40 \mathrm{X}$ en LL y $0.386 \pm 0.01$ vs $0.320 \pm 0.06$ en MCR respectivamente) respecto al grupo sham. La infección miocárdica con AdECA2 o AdGFP no modificó la MCR respecto a lo observado en las ratas GB (tabla 1). Mientras que la $M C$ fue significativamente menor en las ratas LL y BN con AdECA2 respecto a las ratas GB y AdGFP (Tabla 1).

\section{Hipertrofia cardiomiocitaria.}

Área de los cardiomiocitos. La HTA aumentó significativamente el área de los cardiomiocitos en las ratas LL y BN respecto a sus controles sham $(217 \pm 2$ vs $203 \pm 1$ y $222 \pm 6$ vs $180 \pm 5$, respectivamente, figura $1 \mathrm{~A}$ y 1B). La infección miocárdica con AdECA2 disminuyó significativamente el área de los cardiomiocitos respecto al grupo GB respectivo, tanto en ratas LL y BN (-25\%, respectivamente, figuras $1 \mathrm{~B}, 1 \mathrm{C}$ y 2$)$. Los grupos experimentales correspondientes a los controles de infección con GFP no mostraron diferencias en el área cardiomiocitaria respecto a las ratas hipertensas (figuras 1B, 1D y 2) como tampoco por efecto del polimorfismo de la ECA.

Perímetro de los cardiomiocitos. En las ratas GB LL y BN se observó un aumento significativo del perímetro de los cardiomiocitos respecto a sus controles sham $(2 \%, 9 \%$, respectivamente, figura 3$)$. La sobreexpresión de ECA2 en ratas hipertensas LL y BN disminuyó significativamente el perímetro respecto a las ratas GB $(-17 \%,-18 \%$, respectivamente, figura 3$)$. Aunque los perímetros de las ratas LL y BN infectadas con GFP mostraron ser menores que las ratas GB LL y BN (-8\%, en ambos casos), este efecto no logró diferencias estadísticamente significativas. 


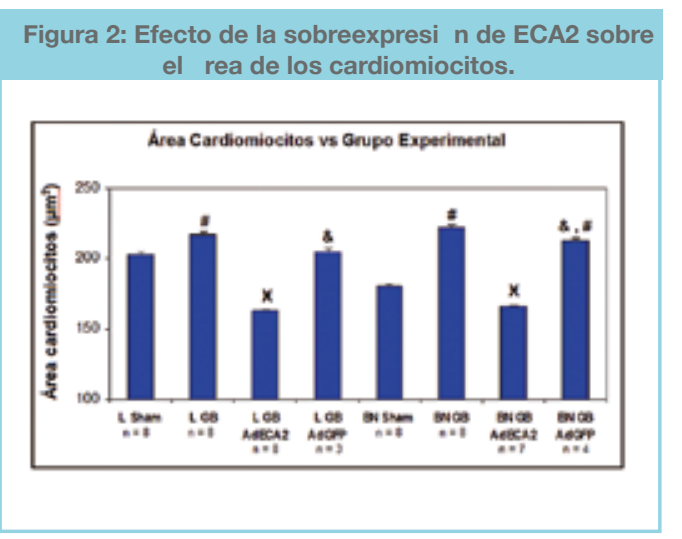

Se usaron ratas hipertensas por sobrecarga de presión LL y BN con niveles genéticamente determinados de ECA y Ang II. El área de los cardiomiocitos se midió por morfometría, tal como se describe en materiales y métodos. Los valores se presentan como promedio \pm SEM. $\square<<0.05$ vs $S, G B, \# p<0.05$ vs $S, \& p<0.05$ vs AdECA2.

\section{Fibrosis Cardíaca.}

Las ratas hipertensas LL y BN sin tratamiento aumentaron significativamente el contenido de colágeno con respecto a las ratas controles sham LL y BN $(6.5 \pm 0.8$ vs $3.7 \pm 0.4$ y $5.9 \pm 0.6$ vs $2.9 \pm 0.3$, respectivamente, Tabla 2). En las ratas BN AdECA2, el contenido de colágeno cardíaco total mostró una clara tendencia a la disminución con respecto a las ratas hipertensas sin tratamiento $(4.6 \pm 0.5$ vs $5.9 \pm 0.6$, respectivamente, tabla 2), disminución que si fue significativa en las ratas LL AdECA2 (3.6 \pm 1.7 vs $6.5 \pm 2.4 \%$ respectivamente, Tabla 2). El contenido de colágeno en las ratas AdGFP fue similar a lo obtenido en las ratas GB, sin efecto del polimorfismo de la ECA.

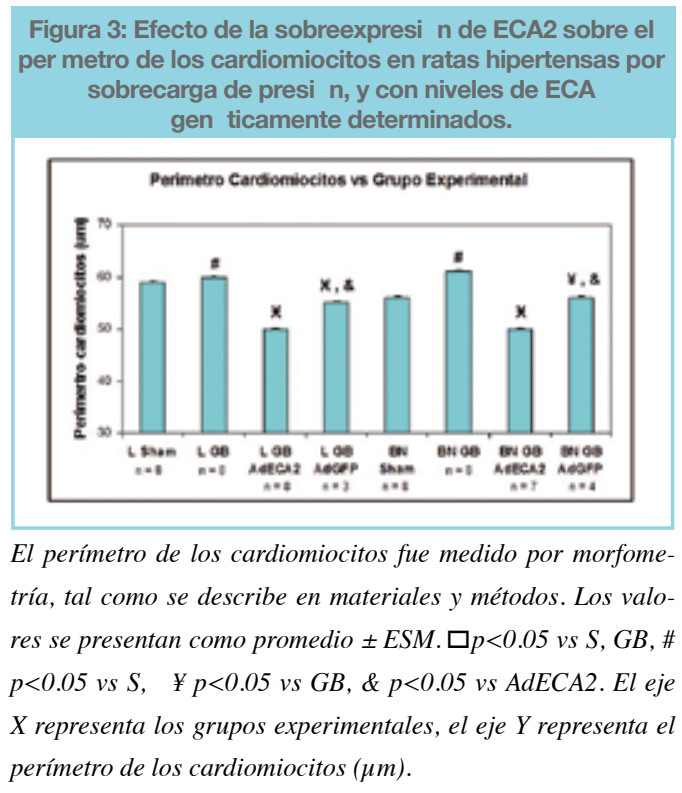

\section{Discusión:}

Nuestros resultados muestran que la sobreexpresión del gen de la ECA2 en ratas con niveles genéticamente altos y bajos de ECA y Ang II y sometidas a un estímulo hipertensivo por sobrecarga de presión: 1) Disminuyó significativamente la PAS, 2) Revirtió el aumento de la masa cardíaca y la hipertrofia del cardiomiocito, y 3) Regresó el incremento del colágeno cardíaco.

El rol potencial de la ECA2 en la HTA está basado en una serie de observaciones experimentales realizadas en modelos genéticos de HTA. Al respecto, Crackower et al. [8] encontraron en ratas Sabra, un modelo de ratas hipertensas sensibles a sal (SBH/y), como también en ratas espontáneamente hipertensas (SHR) y SHR

\begin{tabular}{|c|c|c|c|c|c|c|c|c|}
\hline & $\begin{array}{l}\text { LL-S } \\
(n=8)\end{array}$ & $\begin{array}{l}\text { LL-GB } \\
(n=8)\end{array}$ & $\begin{array}{c}\text { LL-GB- } \\
\text { AdECA2 } \\
(n=4)\end{array}$ & $\begin{array}{l}\text { LL-GB- } \\
\text { AdGFP } \\
(n=3)\end{array}$ & $\begin{array}{l}\text { BN-S } \\
(n=8)\end{array}$ & $\begin{array}{c}\text { BN-GB } \\
(n=8)\end{array}$ & $\begin{array}{c}\text { BN-GB- } \\
\text { AdECA2 } \\
(n=4)\end{array}$ & $\begin{array}{c}\text { BN-GB- } \\
\text { AdGFP } \\
(n=3)\end{array}$ \\
\hline CM & $2.0(0.0)$ & 3.5(0.5)\# & $1.6(0.1) \&$ & $3.2(0.8)$ & $1.4(0.2)$ & $2.5(0.3) \#$ & 2.5 (0.3)\# & $2.9(0.8)$ \\
\hline CS & $1.7(0.2)$ & $3.0(0.4) \#$ & $1.9(0.1)$ & 4.6(1.6)\# & $1.5(0.2)$ & 3.1(0.3)\# & $1.9(0.2) \&$ & $2.8(1.0)$ \\
\hline CT & $3.7(0.4)$ & $6.5(0.8) \#$ & $3.8(0.7) \&$ & 7.7(2.3)\# & $2.9(0.3)$ & $5.9(0.6) \#$ & $4.6(0.5) \#$ & $5.4(1.9)$ \\
\hline
\end{tabular}

Los resultados se presentan como Promedio \pm SEM. Abreviaciones: CM: contenido de colágeno miocárdico (\%), CS: contenido de colágeno sub endocárdico (\%), CT: contenido de colágeno total (\%). \# $p<0.05$ vs $S, \& p<0.05$ vs $G B$. 
propensas a accidente vascular encefálico (SHRSP) que desarrollan HTA sin necesidad de un estímulo hipertensivo, una disminución del mRNA y de la proteína para ECA2, respecto a ratas normotensas (SBN/y) y Wistar Kyoto (WKY). La segunda observación que apoya una relación entre ECA2 y HTA fue también realizada por Crackower et al. ${ }^{8}$, quienes encontraron que el gen de la ECA2 se localiza en el cromosoma X, locus QT relacionado con susceptibilidad a HTA detectado en ratas SBH/y, SHR y SHRSP. Una tercera observación relevante fue hecha por Allred et al. ${ }^{19}$ en ratones ACE2-/- cuya PA basal fue $10 \mathrm{mmHg}$ más alta que ratones normales (ACE2+/y). Igual efecto en la PA se observó en ratones ACE2-/- post administración iv de Ang II. Sin embargo, estos resultados no fueron encontrados por Crackower et al. ${ }^{8}$, ya que ratones ACE2/- no mostraron mayor PA en relación a su grupo control ACE2+/y, aún cuando estos animales mostraron un aumento en los niveles de Ang II en riñón, corazón y plasma. Además, dos polimorfismos en el gen de la ECA2 se han asociado con enfermedad coronaria en humanos ${ }^{8}$.

Para estudiar la hipótesis que la ECA2 cumple un papel en la HTA, Yagil et al. ${ }^{20}$ clonaron el gen de la ECA2 en la región del cromosoma $\mathrm{X}$ adyacente al sitio QTL asociado a HTA. Los autores encontraron un nuevo mecanismo para la HTA en el cual: 1) la ECA2 regula el tono vascular contrarrestando la actividad de la ECA; 2) la menor expresión de ECA2 se asoció con menores niveles de $\mathrm{Ang}_{-}{ }^{1-7}$ en $\mathrm{SBH} / \mathrm{y}$, dejando que la actividad de la ECA2 predomine y aumente el tono vascular y en consecuencia la HTA durante el consumo de sal; y 3) mayor expresión de ECA2 en SBN/y aumenta los niveles de Ang-1-7 y disminuye los niveles de Ang II, previniendo el aumento de la presión sanguínea durante el consumo de sal permitiendo un efecto protector el cual mantiene la normotensión. Por lo tanto, este nuevo mecanismo indicaría que la ECA2 sería una proteína que podría prevenir el desarrollo de HTA.

Una contribución importante y muy reciente que apoyaría que la ECA2 podría ser un blanco terapéutico para el tratamiento de la HTA, ha sido entregado por Diez-Freire et al. ${ }^{21}$, al sobre-expresar el gen de la ECA2 en ratas SHR y WKY mediante la administración intra-miocárdica del vector lentiviral de la ECA2 de murino. La trasferencia génica de la ECA2, mostró una disminución significativa de la PAS desde 180 \pm 9 $\mathrm{mmHg}$ a $149 \pm 2(\mathrm{p}<0,01)$.

La mayoría de la información existente a la fecha respecto al participación de la ECA2 en los mecanismos de homeostasis cardiovascular en la HTA ha sido realizada en modelos genéticos como: ratas espontáneamente hipertensas, ratones ACE2-/- y ratas que sobre expresan el gen de la ECA2. Sin embargo, faltan estudios que evalúen el rol de la ECA2 en procesos fisiopatológicos de remodelamiento cardiovascular, disfunción diastólica y alteraciones en el tono vascular asociado al desarrollo de la HTA, en los cuales la vía clásica del SRA está activa. Al respecto, nuestros resultados en un modelo de HTA por sobrecarga de presión mostraron que la sobreexpresión de ECA2 disminuyó significativamente y tempranamente la PAS de ratas con HTA establecida por 5 semanas post coartación de la arteria renal y los niveles genéticamente determinados de ECA y Ang II no tuvieron un efecto en la respuesta antihipertensiva de la ECA2. Aunque datos preliminares muestran que la infección con adeno GFP disminuyó la PAS en las ratas BN y LL pensamos que los efectos del procedimiento quirúrgicoaltamente invasivo ${ }^{17}$ - pudieran estar influyendo en la medición de la presión. Probablemente es necesario realizar estas mediciones en un tiempo mas alejado de la cirugía para lo cual sería necesario sobre-expresar le ECA2 usando como vector de expresión un lentivirus que permite manejar las sobre-expresiones génicas por tiempos mas prolongados.

En la HTA, el RM se asocia a un aumento de la masa del VI (hipertrofia ventricular izquierda o HVI, alteraciones de la reserva coronaria y re-polarización ventricular prolongada ${ }^{22,23}$. Inicialmente, el proceso de remodelamiento actúa como un mecanismo compensatorio para mantener la funcionalidad del corazón. Sin embargo, los cambios estructurales contribuyen eventualmente a desarrollo de síntomas congestivos por sobrecarga y exacerbación de la disfunción del VI ${ }^{24}$. La disfunción diastólica y sistólica ventricular constituyen la manifestación funcional del remodelamiento estructural adverso del miocardio. El RM se caracteriza, entre otros aspectos, por el aumento de tamaño de los cardiomiocitos y por el desarrollo de fibrosis a nivel intersticial (o fibrosis reactiva) en respuesta a estímulos tales como el aumento sostenido de la presión $\operatorname{arterial}^{2,3}$.

Existen varios reportes en la literatura sobre la participación de ECA2 en la protección de hipertrofia y fibrosis miocárdica. Al respecto, Huentelman et al. ${ }^{7}$ mostraron en ratas a las que se administró un vector lentiviral (intra-miocárdico) que sobre-expresaba ECA2 (Rlenti ECA2+) y posteriormente se les ad- 
ministró Ang II o suero salino por 4 semanas. Como controles se usaron ratas sometidas a un procedimiento experimental similar pero infectadas con un vector lentiviral que sobre-expresaba la proteína fluorescente verde (GFP, Rlenti GFP). La administración de Ang II aumentó significativamente la PA tanto en Rlenti ECA2 y en Rlenti GFP y la sobreexpresión de ECA2 disminuyó significativamente el desarrollo de hipertrofia ventricular y fibrosis miocárdica inducidas por Ang II. Posteriormente, por Diez-Freire et al. ${ }^{21}$, sobre-expresaron el gen de la ECA2 en ratas espontáneamente hipertensas (SHR) y controles Wistar Kyoto (WKY) mediante la administración intra-miocárdica del vector lentiviral de la ECA2 de murino. La transferencia génica de la ECA2 mostró una disminución del $18 \%$ en el grosor de la pared ventricular izquierda, como en la fibrosis perivascular en las ratas SHR-lentiECA2 vs ratas controles infectadas con lentivirus que sobre-expresan la proteína fluorescente verde (GFP). Además, se encontró un aumento del $12 \%$ y $21 \%$ en el diámetro ventricular izquierdo de fin de diástole y sístole, respectivamente. Mientras que las ratas WKY en iguales condiciones experimentales no mostraron diferencias. Recientemente, Raizada y colaboradores usaron un lentivirus ECA2 para prevenir la fibrosis y la hipertrofia miocárdica en ratas SHR y el RM post infarto al miocardio ${ }^{11}$ y Yamamoto et al. ${ }^{9}$ encontraron que la deleción del gen de la ECA2 aumentó la disfunción cardíaca inducida por sobrecarga de presión y el RM asociado con el aumento de los niveles de Ang II y activación del receptor tipo 1 de Ang II.

Nuestros resultados muestran que la sobreexpresión adenoviral de la ECA2 disminuyó la hipertrofia al disminuir tanto el peso cardíaco como el área y perímetro de los cardiomiocitos. Sin embargo, la MCR aumentó significativamente respecto a sus controles producto probablemente a que la infección adenoviral disminuyó significativamente el PC, tanto en ratas infectadas con AdECA2 como con AdGFP. Además, la sobreexpresión de ECA2 regresó la fibrosis cardíaca en el modelo de HTA dependiente de Ang II, siendo la regresión de la fibrosis cardíaca mayor en las ratas LL AdECA2 respecto de las ratas BN.

El modelo de ratas homocigotas LL y $\mathrm{BN}$ ha sido ampliamente utilizado en investigaciones previas del laboratorio ${ }^{14,15,16}$ describiendo que en condiciones basales, las ratas $\mathrm{BN}$ presentan mayores niveles de ECA, renina y Ang II pero baja actividad de NEP y Ang$(1-7)^{13,14}$. En cambio, las ratas LL presentan características opuestas. Por otra parte, el pinzamiento de la arteria renal izquierda (modelo Goldblatt, GB, $2 \mathrm{~K}-1 \mathrm{C}$ ) genera mayor HTA en las ratas BN que las LL [15] y mayor desarrollo de fibrosis miocárdica inducida por isoproterenol ${ }^{16}$, lo cual explica que la regresión de la fibrosis haya sido mayor en las ratas LL tratadas con AdECA2.

Las principales limitaciones de este estudio es que desconocemos a la fecha si el efecto terapéutico de la ECA2 en la disminución de la HTA y la regresión de la hipertrofia y la fibrosis cardíaca se relaciona con mejoría de la función ventricular. Tampoco conocemos las señales celulares gatilladas por la sobre-expresión de ECA2 miocárdica que previenen la HVI y la fibrosis hipertensiva.

Todos los antecedentes disponibles a la fecha muestran el rol de la ECA2 en la prevención de la HTA y el RM isquémico, sobrecarga de presión o aumento de Ang II. En conclusión, nuestros resultados muestran los primeros antecedentes del papel de la ECA2 en la regresión del RM y la disminución de la HTA establecida y apuntan al efecto terapéutico de la sobreexpresión de la ECA2. La modulación de los niveles de la expresión y actividad de la ECA2 mediante terapia génica, podría constituir nuevos blancos farmacológicos para futuras terapias destinadas al control de la HTA y sus complicaciones.

\section{Agradecimientos:}

Este trabajo fue financiado Fondecyt 1070662 


\section{Referencias:}

1. Katovich MJ, Grobe JL, Huentelman M, Raizada MK. Angiotensin-converting enzyme 2 as a novel target for gene therapy for hypertension. Exp. Physiol 2005; 90: 299-305.

2. Mulvany MJ. Effects of angiotensina converting enzyme inhibition on vascular remodelling of resistance vessels in hypertensive patients. J Hypertension Suppl 1996; 14: S21-S24.

3. Varagic J, Frohlich E. Local rennin-angiotensin system: Hypertension and cardiac failure. $\mathrm{J}$ of molecular and cellular cardiology 2002; 34: 1435-1442.

4. Fyhrquist F, Saijonmaa O. Renin-angiotensin system revisited. J Intern Med 2008; 264:224-236

5. Donoghue M, Hsieh F, Baronas E, Godbout K, Gosselin M, Stagliano N, et al. A novel angiotensin converting enzyme related carboxypeptidase (ACE2) converts angiotensin I to angiotensin 1-9. Circ Res 2000; 87: e1-e9.

6. Hamming I, Cooper ME, Haagmans BL, Hooper NM, Korstanje R, Osterhaus A, et al. The emerging role of ACE in physiology and disease. J Pathol 2007;212:1-11

7. Huentelman MJ, Grobe JL, Vazquez J, Stewart JM, Mecca AP, Katovich MJ, et al. Protection from angiotensinII induced cardiac hypertrophy and fibrosis by systemic lentiviral delivery of ACE2 in rats. Exp Physiol 2005; 90: 783-790.

8. Crackower MA, Sarao R, Oudit GY, Yagil C, Kozieradzki I, Scanga SE, et al. Angiotensin-converting enzyme 2 is an essential regulator of heart function. Nature 2002; 417:822-828

9. Yamamoto K, Ohishi M, Katsuya T, Ito N, Ikushima M, Kaibe $\mathrm{M}$, et al. Deletion of angiotensin-converting enzyme 2 accelerates pressure overload-induced cardiac dysfunction by increasing local angiotensin II. Hypertension 2006; 47:718-726

10. Grobe J1, Der Sarkissian S, Stewart JM, Meszaros JG, Raizada MK, Katovich MJ. ACE2 overexpression inhibits hypoxia-induced collagen production by cardiac fibroblasts. Clin Sci 2007; 113: 357-364

11. Der Sarkissian S, Grobe JL, Yuan L, Narielwala DR, Walter GA, Katovich MJ, et al. Cardiac Overexpression of Angiotensin Converting Enzyme 2 Protects the Heart From Ischemia-Induced Pathophysiology. Hypertension 2008; 51:712-718

12. Rivera P, Ocaranza MP, Lavandero S, Jalil JE. Rho Kinase Activation and Gene Expression Related to Vascular Remodeling in Normotensive Rats With High Angiotensin I Converting Enzyme Levels. J Hypertension 2007; 50: $792-$
798

13. Ocaranza MP, Godoy I, Jalil JE, Varas M, Collantes P, Pinto $\mathrm{M}$, et al. Enalalpril attenuates downregulation of angiotensin-converting enzyme 2 in the late phase of ventricular dysfunction in myocardial infracted rat. Hypertension 2006, 48: 572-578.

14. Oliveri C, Ocaranza Mp, Campos X, Lavandero S, Jalil J. Angiotensin I-converting enzyme modulates neutral endopeptidase activity in the rat. Hypertension 2001; 38: 650-654.

15. Ocaranza MP, Piddo AM, Faúndez P, Lavandero S, Jalil JE. Angiotensin I-converting enzyme gene polymorphism influences chronic hypertensive response in the rat Goldblatt model. J Hypertension 2002; 20:413-420.

16. Ocaranza MP, Díaz-Araya G, Carreño JE, Muñoz D, Riveros JP, Jalil JE, et al. Polymorphism in gene coding for ACE determines different development of myocardial fibrosis in rats. Am J Physiol Heart Circ Physiol. 2004; 286: H498-H506.

17. Hajjar RJ, del Monte F, Matsui T, Rosenzweig A. Prospects for Gene Therapy for Heart Failure. Circ. Res 2000; 86; 616-621

18. Nakamura K, Fushimi K, Kouchi H, Mihara K, Miyazaki M, Ohe T, et al. Inhibitory Effects of Antioxidants on Neonatal Rat Cardiac Myocyte Hypertrophy Induced by Tumor Necrosis Factor-a and Angiotensin II. Circulation 1998; 98: 794-799.

19. Allred A, Donoghue M, Acton S, Coffman T. Regulation of blood pressure by angiotensin converting enzyme homologue ACE2. Paper presented at: 35th annual meeting of the American Society of Nephrology 2002, Abstract.

20. Yagil Y, Yagil CH, Crackower M, Penninger J. The protective role of ACE2 in hypertension. Am J Hyper 2003; 16: $23 \mathrm{~A}$.

21. Díez-Freire C, Vázquez J, Correa de Adjounian MF, Ferrari MF, Yuan L, Silver X, et al. ACE2 gene transfer attenuates hypertension linked pathophysiological changes in the SHR. Physiol. Genomics 2006; 27: 12-19.

22. Frohlich ED, Sasaki O, Chien Y, Arita M. Changes in cardiovascular mass, left ventricular pumping ability and aortic distensibility after calcium antagonists in Wistar-Kyoto and spontaneously hypertensive rats. J Hypertens 1992; 10: $1369-1378$

23. Leonetti G, Cuspidi C. The heart and vascular changes in hypertension. J Hypertens Suppl 1995; 13: S29-34.

24. Davis M. A macro and micro view of coronary vascular insult in ischemic heart disease. Circulation 1990; 82: 38-46. 